\title{
Why Place and Voice are different: \\ Constraint-specific alternations in Optimality Theory*
}

\author{
Linda Lombardi \\ University of Maryland, College Park
}

Appeared in Lombardi, L., ed., Segmental phonology in Optimality Theory: Constraints and Representations, Cambridge University Press, 2001.

This version differs slightly from the published text due to rewordings introduced in copyediting.

\section{Introduction}

Both Place and Laryngeal features are often restricted in coda position. The laryngeal distinctions of voicing, aspiration and glottalization are often neutralized to plain voiceless in coda position. The phonology of Place often shows the Coda Condition pattern of Ito (1986), where syllables cannot end in a consonant other than the nasal of a homorganic NC cluster or the first half of a geminate. This is analyzed by Ito as due to a constraint against singly linked Place in a coda.

Both laryngeal neutralization and the alternations involving place have been seen as the result of similar restrictions: constraints that restrict features from appearing in coda position, whether these constraints are negative (as in Ito 1986) or positive (as in Lombardi 1991, 1995a, and the Alignment analysis of the Coda Condition in Ito and Mester 1994). However, the types of alternations that languages exhibit in association with these coda restrictions differ. Although both Laryngeal Constraint and Coda Condition languages may show codas in assimilated clusters escaping the constraint, beyond that there seem to be more differences than similarities in the behavior of Place and Voice. Both types of features may be subject to neutralization. But coda restrictions on Place may result in either epenthesis or deletion, while forbidden coda Voice never triggers these alternations. ${ }^{1}$

In a theory where phonological rules specify both context and change, as in SPE and much work following upon it, it is not possible to account for this asymmetry of patterns except by stipulation. For example, an autosegmental rule deleting a voiced consonant from coda 
position is as well formed as a rule deleting a consonant with Place from the coda, even though the former is unattested. But since it is possible to write all the rules that do exist with the machinery of the theory, we can easily construct analyses of any given language, and the gaps in the cross-linguistic typology are not made obvious.

A theory that tries to combine constraints and rules runs into similar difficulty. For example, Lombardi (1991, 1995a), following Mester and Ito (1989), suggests that Laryngeal neutralization is an automatic consequence of the violation of a constraint that rules out Laryngeal features except in a certain position: delinking the feature repairs the violation without requiring any additional information. However, delinking is not an automatic consequence of having Place in coda; either epenthesis or deletion are also possible. Delinking may be the universal reaction to Laryngeal violations, but it is not the universal reaction to Place violations; and vice-versa, the other possible responses to Place violations do not occur in response to voicing. Thus we must stipulate that certain repairs can be connected only to certain constraints.

Optimality Theory (Prince and Smolensky 1993) focusses our attention on this kind of asymmetry for two reasons. One is that it is not possible even to stipulate a connection between particular constraints and associated alternations. Since we cannot avoid the problem by stipulation, we are forced to look for a more satisfactory approach. Second, it is not possible to ignore the problem of the asymmetry in OT because cross-linguistic typology is absolutely central to the theory. One fundamental premise of OT is that UG consists of a set of constraints and that languages differ only in how they rank these constraints; all language-particular variation comes from the different effects of constraint interactions in different rankings. Thus, in order to argue that a certain set of constraints exists, we must show that rerankings of these constraints give all and only attested grammars. If rerankings do not give the appropriate cross-linguistic typology, the proposed constraints must not be the correct analysis of the phenomena in question.

For instance, suppose there is a Coda Condition on Place and we can rank it above the anti-insertion constraint so that epenthesis is optimal. If there is a similar coda constraint on Voice, the same ranking should be possible, incorrectly predicting the possibility of epenthesis. We cannot stipulate that the connection between the voicing constraint and the epenthetic repair is impossible. Thus, this first, most obvious attempt at a constraint-based analysis - that both features are subject to a Coda Condition - must be incorrect; we are forced to find an alternate account that makes the correct typological predictions. 
I will argue in this paper that if we propose that Universal Grammar contains the appropriate constraints and representational properties, Optimality Theory can provide an appropriately restrictive account of the alternations involving Place and Voice. The analysis will be based on the following substantive claims:

1. Although both types of features are subject to position-independent markedness constraints like *Voice, *Dor, only Place is subject to a positional markedness constraint, CodaCond.

2. There are consonants that are not marked with any Laryngeal specification, but none that are truly Placeless: /h, $7 /$ are marked with Pharyngeal place and are subject to a low-ranked Place markedness constraint. This will not only solve part of the problem regarding the differences in alternations, but is important with respect to longstanding problems with the representation of $/ \mathrm{h} /$ and $/ R /$.

3. It is necessary to recognize the existence of MaxFeature constraints that ensure realization of underlying feature specification, as suggested in McCarthy and Prince (1995).

The particulars of the analysis are important for featural phonology in general since they speak to issues of neutralization, markedness, and faithfulness that are relevant for the treatment of features other than Place and Laryngeal. However, the results also have broad implications for other cases where we see similar asymmetries and limits on the attested types of alternations associated with a constraint.

\section{Differences between Place and Voice: the data}

In this section I will review the data that shows the different patterns of alternations in response to Place and Voice in coda position.

\subsection{Laryngeal neutralization}

In many languages, syllable-final consonants cannot bear the laryngeal distinctions of voicing, aspiration, or glottalization. For the present purpose the most interesting cases are those that show active alternations, such as the well- known case of syllable-final devoicing in German: 
(1) German: syllable-final laryngeal neutralization

a. /rund/

run[d]e 'round plu.'

run[t] 'round sng. '

Run[tv]orm 'roundworm' /veg/

We[g]e 'way - pl.'

We[k] 'way - nom.'

We[kš]necke 'slug(path+snail)'

In German, syllables may not end in a voiced consonant. If the final consonant of a morpheme like/veg/ becomes syllable-final at the surface, it is devoiced. See Lombardi (1991, 1995a) for additional data.

\subsection{Coda Condition on Place}

It is also possible for a language to forbid Place features in a coda. In the analysis of Ito (1986, 1989), geminates and place-assimilated clusters escape the Coda Condition on Place because there is not a single link from the coda position to a Place feature; Ito and Mester (1994) discuss how to analyze this effect in OT by refining the definition of Alignment. But what I am most concerned with in this paper is what happens when such double linking does not exist: what happens where the consonant cannot have its own Place, but also does not come to share Place with the following consonant?

The alternations triggered by the coda constraint on Place are more varied than those triggered by the inability to have coda Voice. Ito gives examples of two: epenthesis of a vowel (making the consonant syllable-initial)and deletion of the offending consonant. I will give examples of these, and then turn to the possibility of coda Place neutralization.

\subsubsection{Epenthesis}

In Ponapean (Rehg 1984, Rehg and Sohl 1981) word-internal syllables are either open, closed by the first segment of a geminate, or closed by a nasal in a homorganic cluster:

\section{(2) Ponapean syllable types}

pereki 'to order'

lusida 'to jump upwards'

nampar 'trade wind season' urenna 'lobster'

rerrer 'to be trembling' 
When two consonants come together by morpheme concatenation, various alternations ensure that these syllable constraints are met. Nasals assimilate in Place, and in some cases there is nasal substitution, creating homorganic nasal- stop clusters. In the absence of the environment for those processes, a vowel is epenthesized, making the consonant syllable-initial. (The quality of the vowel is different in different circumstances, but is predictable.) Word-final consonants escape the Coda Condition by final extraprosodicity. ${ }^{2}$

\section{(3) Ponapean epenthesis}

/kitik men/ kitikimen 'rat'

/ak dei/ akedei

/ak tantat/ akatantat (R\&S p 92; no glosses) reduplicated:

net netenet 'smell'

tep tepitep 'start'

kos kosokos 'throw'

Ito's analysis of this set of facts brings them all together as the result of a prohibition on Place syllable-finally:

(4) Coda Condition (CodaCond):

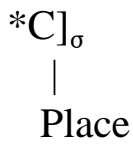

Doubly linked structures such as Place-assimilated clusters and geminates will escape this constraint; for other consonants, epenthesis renders them syllable-initial, so the constraint is not violated.

Ito (1986) also discusses alternations in Sino-Japanese compounds, where the facts are essentially the same except for the absence of final extraprosodicity; another case is Axininca Campa (Payne 1981; except for person prefixes).

\subsubsection{Deletion}

Diola (Sapir 1965) deletion is analyzed by Ito as triggered by the coda condition on Place, with deletion of unlicensed consonants following from Stray Erasure as first suggested by Steriade (1982) in her analysis of this language. Like Ponapean, Diola allows homorganic nasal-stop clusters and geminates, but aside from this there are no coda consonants. Nasals may be preserved by assimilating to the following consonant; but where assimilation is inapplicable, the coda consonant deletes ${ }^{3}$ : 


\section{(5) Diola consonant deletion}

$\begin{array}{lll}/ \text { let+ku+jaw/ } & \text { lekujaw } & \text { 'they won't go' } \\ \text { /ujuk+ja/ } & \text { ujuja } & \text { 'if you see' } \\ \text { /-kob kob en/ } & \text { kokoben } & \text { 'yearn' }\end{array}$

Additional cases of this type are Akan (Schachter and Fromkin 1968) and Axininca (Payne 1981; person prefixes).

\subsubsection{Place neutralization}

A final pattern that disallows Place distinctions in coda position is neutralization to an unmarked Place. For example, in Slave (Athabaskan; Rice 1989) all nonsonorant consonants surface as /h/ when syllable-final.

\section{(6) Slave syllable-final Place neutralization}

\begin{tabular}{|c|c|c|c|c|}
\hline /ts'ad/ & $\begin{array}{l}\text { ts'ah } \\
\text { ts'ade }\end{array}$ & 'hat' & /xaz/ & $\begin{array}{l}\text { xah } \\
-y a z e\end{array}$ \\
\hline /seey/ & $\begin{array}{l}\text { seeh } \\
\text {-zeeye }\end{array}$ & 'saliva' & /tl'ut/ & $\begin{array}{l}\text { tl'uh } \\
\text {-tl'ute }\end{array}$ \\
\hline
\end{tabular}

Sonorants are treated differently, but also do not allow Place distinctions in coda: Nasals delete and nasalize the preceding vowel, and the only possible syllable-final non-nasal sonorant is /y/.

Another example is the Kelantan dialect of Malay (Teoh 1988), where / ktp/ become / $/$ in coda position and /s/ (and borrowed /f/) become /h/.

(7) Kelantan Malay

/ikat/ ika? "tie"
/dakap/ daka? "embrace"
/tapis/ taplh "to filter"

(As in Slave, sonorants are also treated differently, but the result is that there are also no Place distinctions in coda.) 
As we see, in both of these languages, codas neutralize to some segment that has an unmarked Place. Other distinctions may be possible in coda - the sonorant/obstruent distinction in Slave, the stop/fricative distinction in Malay - but Place distinctions are not: corresponding to the obstruents, we see only $/ \mathrm{h}, \mathrm{l} /$. Neutralization of all distinctions to $/ \mathrm{R} /$ is also possible, as in for example Burmese (Okell 1969), where / $/$ is the only possible coda. I will concentrate here on neutralization to $/ \Omega /$ as the representative case, to simplify comparison of the overall patterns to those for Voice. Additional cases are numerous; some examples are Austronesian languages Selayarese (Mithrun and Basri 1986), Buginese, Makasserese (Mills 1975), and Sangir (Maryott 1961); Macushi (Macuxi; Carib, Abbott 1986, Carson 1982); New Guinea languages Kanite, Kamano, and Fore (Nicholson 1962, Young 1962); Usarufa (Bee and Glasgow 1973); Tucana (West and Welch 1967) and Guanano (Waltz 1967).

\subsection{Summary}

We can sum up the data in the following way. I use hypothetical examples to facilitate comparison. Of the three logical possibilities for avoiding a feature in Coda position, in response to coda Voice we find only the first is attested:

$$
\begin{array}{lll}
\text { /pig/ - > pik, } & \text { /pik/ -> pik } & \text { Neutralization: German } \\
\text { /pig/ -> pigi, } & \text { /pik/ -> pik } & \text { Epenthesis: unattested } \\
\text { /pig/ -> pi, } & \text { /pik/ -> pik } & \text { Deletion: unattested }
\end{array}
$$

In contrast, in response to coda Place we find all three possibilities occur:

$$
\begin{array}{ll}
\text { /pip, pit, pik/ -> pi? } & \text { Neutralization: Slave } \\
\text { /pip, pit, pik/ -> pipi, piti, piki } & \text { Epenthesis: Ponapean } \\
\text { /pip, pit, pik/ -> pi } & \text { Deletion: Diola }
\end{array}
$$

\subsection{Place assimilation}

A final detail should be noted about the facts regarding Place. Assimilation is another way of avoiding violations of the Coda Condition, as shown by Ito (1986). Indeed, unless a language has such assimilation, we have no evidence that CodaCond is active. Deletion and epenthesis 
can also be an effect of NoCoda, which rules out all syllable-final consonants. We only have evidence for active CodaCond when a language has the pattern described by Ito (1986) where syllables closed by the first half of geminates and/or assimilated NC clusters are permitted.

This means that the languages relevant to the consideration of Place phonology all have certain types of assimilation, in addition to the alternations that I will concentrate on. There are some environments where Place or total assimilation take place; outside of these environments, then, is where we see the other types of alternations forced by the need to satisfy CodaCond.

A detailed analysis of Place assimilation is outside the scope of this paper. (See Padgett 1995a for an approach.) So except for Place neutralization languages like Slave, where assimilation is not an issue, this means that I will be giving only a partial analysis of coda Place phonology for a given language. Consider the contrast between nasal-stop clusters and all other clusters in Diola, for example. Nasals are obviously the consonants most susceptible to assimilation and any analysis of Place assimilation will need to account for this. Perhaps there are separately ranked constraints triggering assimilation for stops and nasals; perhaps there are differently ranked Faithfulness constraints for stop and nasal Place. Either way, we simply need to concentrate on those cases where the consonants are non-nasal and we will be able to see the interactions of the other constraints, which are the focus of interest in this paper.

\subsection{Outline}

In section 3 I will present the analysis of Lombardi (1999) of laryngeal neutralization. I will then explain how this analysis can be used to explain why we do not see epenthesis or deletion in response to coda Voice restrictions. Section 4 will argue that the existence of epenthesis in response to coda Place shows that there must be an explicit CodaCondition constraint as in Ito (1986). Section 5 then turns to the question of deletion. I will first show how neutralization of Place can be analyzed with fully Place-specified consonants, therefore arguing that there are no Placeless consonants. Then, I will show how this difference in representation - there are Laryngeally unmarked consonants, since voice is privative - accounts for the existence of deletion in response to Place but not Voice. Section 6 sums up the analysis of the differences in the phonological patterns. 


\section{Laryngeal patterns}

\subsection{Analysis of Lombardi (1999)}

The analysis of Lombardi (1999) first of all assumes privative [voice]: a voiced obstruent is marked Voice, a voiceless obstruent has no Laryngeal specification. This argued for by Lombardi (1991, 1995a,b), Mester and Ito (1989), Cho (1990). We will see that this assumption is crucial to the argument in this paper in section 5.

Central to the analysis in this paper is the Correspondence Theory framework of faithfulness constraints (McCarthy and Prince 1995). I will assume the constraint MaxIOSeg penalizing deletion and DepIOSeg penalizing epenthesis, abbreviated Max and Dep. I will also argue that we must recognize a type of MaxIO constraint that regulates direct correspondence between features, unlike McCarthy and Prince's IdentF family, which only penalizes feature changing, regulated through the segment.

For the analysis of laryngeal neutralization, I will define only the constraints and basic interactions that are crucial to the present arguments. For more detailed discussion of these constraints and others affecting voice assimilation, including the complete factorial typology, see Lombardi (1999).

\section{(10) IdentOnset(Laryngeal) (Abbreviated: IDOnsLar)}

A is a segment in the Input and B in the Output:

If $\mathrm{B}$ is in onset position, it must be faithful to the Laryngeal specification of A.

This member of the Ident family is specified for position: such positional faithfulness constraints are also proposed by Beckman (1995, 1998), and Padgett (1995a) to account for a range of different cases cross-linguistically where certain features have a preference for certain positions, often resulting in neutralization of some kind in other positions. ("Onset" is a shorthand for the released prenuclear position described by the Laryngeal Constraint of Lombardi (1991, 1995a); see Lombardi (1999) for additional discussion. Examples in this paper are chosen so that this simplified version is sufficient.)

Given privative [voice], I assume that even though voiceless consonants have no feature marking, the Ident constraint can tell the difference between voiced and voiceless: a 
segment with either an added Laryngeal feature or a missing one will cause a violation of Ident.

\section{(11) MaxLaryngeal (MaxLar)}

Every Laryngeal autosegment in the input has a correspondent in the output.

As we will see below, this additional type of featural faithfulness will be crucial to the results herein. Although their analyses employ Ident constraints for featural faithfulness, McCarthy and Prince do suggest that MaxFeature constraints may exist, and that Ident may actually be decomposed into MaxF and DepF components (McCarthy and Prince 1995:71). Other authors have used these constraints (LaMontagne and Rice 1995, Causley 1997, Walker 1997) or constraints that have a similar effect (Pater 1995).

(12) *Lar : Don't have Laryngeal features

This is one of the family of featural markedness constraints as proposed by Prince and Smolensky (1993). A consonant bearing a Laryngeal feature violates this constraint; since voice is assumed to be privative, a voiceless consonant does not.

The interaction of $*$ Lar with the positional faithfulness constraint may result in voicing being possible in the onset and not the coda: this is the result of the ranking IDOnsLar >> Lar >> MaxLar. Consider first a simple monosyllable:

(13a) Syllable-final Laryngeal neutralization (German)

\begin{tabular}{|l|c|c|c|}
\hline /veg/ & IDOnsLar & $*$ Lar & MaxLar \\
\hline veg & & $* !$ & \\
\hline 吗ek & & & $*$ \\
\hline
\end{tabular}

\begin{tabular}{|l|c|c|c|}
\hline$/$ der/ & IDOnsLar & $*$ Lar & MaxLar \\
\hline ter & & $*$ & \\
\hline $\mathrm{der}$ & $* !$ & & $*$ \\
\hline
\end{tabular}


As we see from this table, this ranking gives devoicing syllable-finally. The * Lar violation for the syllable-final consonant in [veg] is more serious than the MaxLar violation incurred when the consonant is devoiced; the devoiced consonant has no Laryngeal features, and so does not violate *Lar. Syllable-initially, however, voicing will be maintained, because IDOnsLar outranks *Lar. In the onset it is more important for the correspondent input and output segments to agree in laryngeal specification, as required by IDOnsLar, than it is for them to lack laryngeal marking, as required by $*$ Lar.

If * Lar is ranked above both faithfulness constraints, voicing will be entirely prohibited in obstruents.

(14) Voiceless obstruents only (Hypothetical input)

\begin{tabular}{|l|c|c|c|}
\hline /big/ & $*$ Lar & IDOnsLar & MaxLar \\
\hline mik & & $*$ & $* *$ \\
\hline big & $* ! *$ & & \\
\hline bik & $* !$ & & $*$ \\
\hline pig & $* !$ & $*$ & $*$ \\
\hline
\end{tabular}

As the tableau shows, *Lar gives a mark for each voiced consonant. Thus the candidate with all voiceless consonants will be optimal regardless of faithfulness violations. This give the common type of language that has only plain voiceless obstruents.

Thus the proposed constraints can produce languages that allow voicing in onset but not coda, and languages that have no voiced consonants. In addition, where * Lar is lowest ranked, the result is a language with no voicing distinctions in obstruents. The remaining logically possible pattern - voicing in the coda but not in the onset - is impossible, which is correct, since there are no such languages. As we see, even if we reverse the ranking of the faithfulness constraints there is no ranking that gives this pattern; either voicing is possible everywhere (4), or voicing is prohibited entirely (16). 


\section{(15) Voice unrestricted}

\begin{tabular}{|l|c|c|c|}
\hline$/ \mathrm{big} /$ & MaxLar & $*$ Lar & IDOnsLar \\
\hline pik & $* ! *$ & & $*$ \\
\hline ॠbig & & $* *$ & \\
\hline bik & $* !$ & $*$ & \\
\hline pig & $* !$ & $*$ & $*$ \\
\hline
\end{tabular}

(16) Voice prohibited

\begin{tabular}{|l|c|c|c|}
\hline$/$ big/ & $*$ Lar & MaxLar & IDOnsLar \\
\hline big & & $* *$ & $*$ \\
\hline bik & $* ! *$ & & \\
\hline pig & $* !$ & $*$ & \\
\hline
\end{tabular}

These constraints generate the correct typology without a specific constraint against laryngeal features that mentions the syllable-final position. Neither is there a licensing constraint as in Lombardi $(1991,1995 \mathrm{a}, 1995 \mathrm{c})$ that describes the licensed position. There is a constraint that mentions position, but it is the faithfulness constraint: Onset is the position where it is important to be faithful. The constraint that may trigger devoicing is simply the markedness constraint *Lar, needed anyway to account for the relative markedness of voiced and voiceless consonants. See Lombardi (1995b) for more discussion, where it is shown that this approach gives the correct typology of voicing assimilation and neutralization. ${ }^{4}$

Thus, under this analysis, devoicing is a result of markedness of [voice] interacting with positional faithfulness constraints. There is no specific licensing or coda-condition type of constraint. As we will see, Place will differ. Although most of the constraints affecting Place are parallel to the ones affecting laryngeal features, the wider variety of Place alternations will be evidence for additional constraints. 


\subsection{MaxLar and Faithfulness "overkill"}

So far this sums up some of the results of Lombardi (1999). Now I will turn to the question of why there is only one possible response to the prohibition on syllable-final voiced consonants. We know that impermissible Place seems to be able to trigger epenthesis (Ponapean) or deletion (Diola). Why does this never happen with impermissible voiced consonants?

Assuming a language with the ranking of laryngeal constraints that causes voiced coda consonants to be impossible, what repair options are possible when an underlying voiced consonant is in danger of being in coda position? The following table shows the violations incurred by the relevant candidates.

(17) No epenthesis or deletion due to *Lar: hypothetical input/pig/

\begin{tabular}{|l|l|l|l|l|}
\hline & $*$ Lar & MaxLar & Dep & Max \\
\hline pig & $*$ & & & \\
\hline pik & & $*$ & & \\
\hline pigi & $*$ & & $*$ & \\
\hline pi & & $*$ & & $*$ \\
\hline
\end{tabular}

As we see by examining these marks, no ranking of Dep and Max with respect to these constraints can cause deletion or epenthesis to be optimal. First of all, in the languages of interest, where *Lar is high ranked, both the faithful [pig] and the epenthesized [pigi] have a fatal *Lar violation; since * Lar applies regardless of the position of the consonant, moving the voiced consonant to an onset does not eliminate the violation. The choice then must be made between [pik] and [pi]. [pi] will never be optimal, no matter how the constraints are ranked, because it has more Faithfulness violations than are necessary to satisfy the markedness constraints. Both [pik] and [pi] violate MaxLar since their laryngeal feature is not parsed, but [pi] has an additional Max violation that [pik] does not have. So Max will make the decision in favor of the devoiced [pik] regardless of where it is ranked.

What we see, then, is that given the proposed constraints and representations, segment deletion will always be "overkill" as a way of satisfying the markedness constraint, and will 
never be optimal. This situation corresponds to that described in Legendre, Raymond and Smolensky's (1993) discussion of the typology of case systems: If the marks of candidate A are a subset of the marks of candidate B, B will never be optimal in any grammar.

Using the MaxF constraint for featural faithfulness is crucial to this result. With Ident, it would be impossible to rule out deletion or epenthesis in response to coda voicing. As seen in the following tableau, if faithfulness to [voice] in non-onset position were governed by an Ident constraint, there would be no featural faithfulness violation in the last candidate, in which the final consonant is deleted:

\begin{tabular}{|l|l|l|l|l|}
\hline /pig/ & $*$ Lar & IDLar & Dep & Max \\
\hline pig & $* !$ & & & \\
\hline pik & & $* !$ & & \\
\hline pigi & $* !$ & & $*$ & \\
\hline pi & & & & $*$ \\
\hline
\end{tabular}

This is due to the definition of McCarthy and Prince's Ident constraints, which refer to correspondent segments. Since/g/ has no output correspondent in the last candidate, it does not violate any Ident constraint for the features of the input.

Without MaxLar, then, we cannot account for the absence of languages where the Laryngeal Constraint is satisfied by deletion: that is, a language with a voicing distinction in obstruents wherein coda voiced consonants delete, but coda voiceless do not. It would be possible to construct a grammar for such a language: It would have the ranking in the tableau above, along with high-ranked Onset to prevent deletion of voiced onset consonants. As we see in the following tableau, this ranking, which deletes voiced coda consonants, would not delete voiceless coda consonants:

(19)

\begin{tabular}{|l|l|l|l|l|}
\hline$/$ pik/ & $*$ Lar & IDLar & Dep & Max \\
\hline pi & & & & \\
\hline pik & & & & $* !$ \\
\hline
\end{tabular}


Such a language is unknown; thus the MaxF constraint is essential. ${ }^{5}$

\section{Place and epenthesis: the need for CodaCond}

Above we have seen why deletion and epenthesis are impossible if we assume the Lombardi (1999) analysis of voicing neutralization. However, these additional patterns are possible for Place. We now turn to the question of what differs about Place that allows this. I will propose that there are two differences. One involves what constraints exist that refer to each type of feature. The other is a representational difference.

First, in this section, I will discuss epenthesis. I will show that the crucial difference here is that there are different constraints affecting the two types of features. I will show that we can account for some of the differences in phonological behavior by recognizing that while both Place and Voice are subject to position-neutral markedness constraints like *Voice and *Dor, only Place is subject to a positional markedness constraint, CodaCond (Ito 1986).

In the next section we turn to deletion. I will show that the difference here turns on a representational difference, based partly on the assumption of privative [voice] made earlier: that plain voiceless consonants have no Laryngeal features at all, but there are no truly Placeless consonants. The latter is a claim that I will show we can make in Optimality Theory, despite the arguments of previous theories that [h,?] must be Placeless.

The analysis of voice neutralization given above uses no constraint that specifically forbids Voice in coda. Rather, the alternation is shown to be a result of the interaction of context free markedness - *Lar - with positional and general faithfulness - IdentOnsLar and MaxLar. So we have seen that some coda effects, abstracting away from the particular feature, can be the result of the following type of constraint interaction:

(20) Onset Feature Faithfulness $>$ *Feature $>>$ General feature faithfulness

For Place features, parallel constraints must exist to account for markedness facts: the family of *F constraints on Place features (e.g. *Dor, *Cor) was proposed in the original work on OT (Prince and Smolensky 1993). Furthermore, in section 5, we will see that the existence of such constraints and of positional faithfulness (IdentOnsetPlace) is crucial to part of the present argument.

So why do we need CodaCond additionally, if for Voice we could analyze coda effects without a constraint referring to codas? 
While it is possible to produce certain of the Place alternations from a markedness/faithfulness interaction parallel to that proposed for laryngeal alternations, the facts about the difference between Place and Voice show that such constraints are not sufficient to account for the phonology of Place. As we saw in section 3, the interaction of faithfulness and context-free markedness will never result in epenthesis as a reaction to a prohibition on a feature in coda. But Place show exactly this kind of alternation.

The solution to this is simple: We must simply assume in OT the existence of the constraint proposed in Ito (1989), repeated here from (4)

\section{(21) CodaCond:}<smiles>[Mg][Mg]Cl</smiles>

Interactions of Dep, Max and CodaCond can give languages where vowel epenthesis is optimal. (I use the same hypothetical input throughout to facilitate comparison.) If Dep is ranked below CodaCond, then a language can avoid violating the coda condition by inserting a vowel:

\begin{tabular}{|l|c|c|}
\hline$/$ pik/ & CodaCond & Dep \\
\hline pik & $* !$ & \\
\hline$\square$ piki & & $*$ \\
\hline
\end{tabular}

The opposite ranking disallows this type of alternation.

\begin{tabular}{|l|c|c|}
\hline$/$ pik/ & Dep & CodaCond \\
\hline pik & & $*$ \\
\hline piki & $* !$ & \\
\hline
\end{tabular}


Max must also be high ranked for epenthesis to be optimal, to rule out the candidate that deletes the offending consonant. For the present purpose then the full ranking is the following.

(24) Languages with epenthesis in response to CodaCond ${ }^{7}$

\begin{tabular}{|l|l|l|l|}
\hline /pik/ & Max & CodaCond & Dep \\
\hline pik & & $* !$ & \\
\hline pi & $* !$ & & \\
\hline 霞iki & & & $*$ \\
\hline
\end{tabular}

Thus, with the addition of CodaCond, we can produce a grammar with the epenthesis alternation. Why does this differ from the result using with positional faithfulness alone?

The markedness constraints give a mark to a consonant whether it is syllable-final or syllable-initial: thus high-ranking markedness will never result in epenthesis. As we saw above with *Voice, both [pig] and [pigi] have a mark for the *F constraint *Dor. Epenthesis does nothing to solve the *Dor problem, so it will never be prompted by place-neutral markedness. This is in clear contrast to the interaction of CodaCond and epenthesis: if Dep is low ranked, epenthesis allows satisfaction of CodaCond:

(25) CodaCond and Markedness effects difference

\begin{tabular}{|l|l|l|}
\hline$/$ pik/ & CodaCond & Dep \\
\hline pik & $*$ & \\
\hline piki & & $*$ \\
\hline
\end{tabular}

\begin{tabular}{|l|l|l|}
\hline$/$ pik/ & $*$ Dor & Dep \\
\hline pik & $*$ & \\
\hline piki & $*$ & $*$ \\
\hline
\end{tabular}

Thus, epenthesis can be optimal when CodaCond is high ranking, since those two candidates differ in their violations of CodaCond; they do not differ in their violations of *Dor, so no ranking of $*$ Dor can motivate epenthesis.

We see, then, that all of the Place constraints are necessary. Although the markedness and faithfulness constraints are independently required, they cannot account for the existence of the epenthesis alternation; therefore CodaCond must exist as well. ${ }^{8}$ 


\section{Neutralization and Deletion with Place coda restriction}

We now turn to the question of why we see deletion in response to coda Place when we do not see it in response to coda Voice. Understanding the nature of the Place-unmarked consonant is crucial to the explanation.

\subsection{Place of $/ 7, \mathrm{~h} /$}

It has often been argued that /h, ?/ are Placeless, based on phenomena such as transparency to spreading (Steriade 1987) and apparent Place delinking such as the cases in 2.2.3. Unfortunately, this proposal is inconsistent with the evidence from languages that show the natural class of gutturals - uvulars, glottals, and pharyngeals - which has been amply documented by McCarthy (1989, 1994a). It has been impossible to reconcile these two approaches in previous theoretical frameworks, forcing the conclusion that different languages differ in their representations of [h,?]: in some they are Placeless, and in some they have Pharyngeal place.

I will suggest that OT allows a resolution of this problem that does not require language-specific representations for these consonants, and that will be crucial to the analysis of the phonological asymmetries described in this paper.

Following Prince and Smolensky (1993) and Smolensky (1993), I assume that Place markedness is not due to underspecification, but rather to a universally ranked family of markedness constraints: so the fact that Coronals are more unmarked than Dorsals and Labials is due to the following universally ranked constraints:

(26) *Dor, *Lab > * Cor

It is proposed that this family of constraints is not freely rerankable by individual languages, but is fixed by UG, since all languages show the same Place markedness relations.

Next, I will assume that the place feature that defines $/ 2, \mathrm{~h} /$ is Pharyngeal, following McCarthy $(1989,1994 a)$. This accounts for the fact that these consonants pattern as part of the natural class of gutturals. In order to distinguish the different Pharyngeal consonants we will call on a dependent Place feature; I follow unpublished work by McCarthy (1989) for concreteness ${ }^{9}$ : 

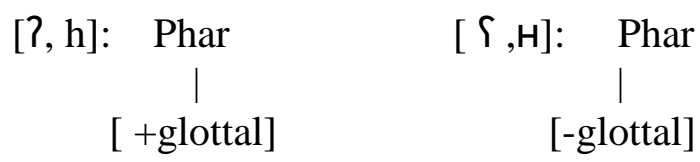

Then, to the hierarchy of markedness constraints I will add Pharyngeal place as the least marked:

(28) Place Hierarchy (PLHier): $\quad$ *Dor, *Lab >> *Cor $>>$ *Phar

Although it may seem odd for Phar to be the least marked place given the cross-linguistic rarity of the true Pharyngeals, I suggest that the markedness of the gutturals must be due not to their primary Place but due to other features or combination of features that they bear; in the simplest case that perhaps [-glottal] is highly marked. We can usefully compare this to a consonant like English /ð/ which is highly marked crosslinguistically despite its relatively low marked Coronal primary Place.

This constraint-ranking approach to markedness allows us to analyze the special behavior of glottals and coronals in epenthesis (Lombardi 1997) and transparency (Gafos and Lombardi 1999). It thus allows a uniform representation for /h, $\mathrm{l} /$ in all languages: they are Phar in languages where they form a natural class with gutturals, and they are also Phar in languages with neutralization, $/ 2 /$ epenthesis, and transparency effects ${ }^{10}$.

For the purposes of this paper, this approach will help explain the remaining differences between Place and Voice phonology. I assume that a well formed consonant must always have Place: in OT terms, that a constraint HavePlace is never violated. But given privative Voice there are truly laryngeally unmarked consonants; there is no constraint HaveLar. This will result in differences in phonological patterns. A glottal stop will get a mark for violating some constraint against Place, even if it is the most unmarked Place; a voiceless unaspirated stop will violate no constraint on laryngeal features. Obviously this will have crucial repercussions.

\subsection{Neutralization}

The assumptions above will allow us to analyze Place neutralization without Placelessness, but rather as a change to the least marked Place. 
I assume faithfulness constraints parallel to those for laryngeal features. IDentOnsPlace requires that an onset consonant have the Place of its input correspondent. MaxPlace requires that an underlying Place feature have an output correspondent. The universal ranking of these constraints, as for laryngeal features, will be:

\section{(29) IDOnsPlace >> MaxPlace}

The interactions of markedness and faithfulness do not have the same freedom for Place as they do for Laryngeal features. Since all languages have Place in consonants at least syllable-initially, the ranking IDOnsPlace >> PLHier must be fixed. This is in contrast to Laryngeal features, where *Lar > IDOnsLar is possible, yielding a language with only plain voiceless consonants.

Since this ranking is fixed, the constraint IDOnsPlace will generally be omitted in the following tableaus. All languages have Place distinctions syllable-initially, and syllable-initial consonants will not be involved in the alternations to be examined.

We can now analyze the loss of Place distinctions in coda, as exemplified by Slave in section 2.2.3. With the constraints proposed so far, Place neutralization can be optimal when MaxPlace is too low ranked to force the original Place feature to be parsed, and Max and Dep are too high for deletion or epenthesis to be an option. McCarthy and Prince's definition of the Max constraint is crucial to the analysis: Max requires that there be some consonant in correspondence with the input consonant, but it does not require that the correspondents agree in all features; thus [?] satisfies Max as a correspondent for the underlying /g/. (*F marks for syllable-initial consonants, which are irrelevant to the outcome, are omitted in the next two tableaus.)

(30) Syllable-final Place neutralization (Malay)

\begin{tabular}{|l|c|l|c|c|c|c|}
\hline /ikat/ & Max & Dep & CodaCon & $*$ Cor & $*$ Phar & MaxPl \\
\hline ikat & & & $*$ & $* !$ & & \\
\hline ikati & & $* !$ & & $*$ & & \\
\hline ika & $* !$ & & & & & $*$ \\
\hline 舟ika? & & & $*$ & & $*$ & $*$ \\
\hline
\end{tabular}


As we see in the tableau, both epenthesis and deletion are ruled out by Max and Dep. Both faithful [ikat] and debuccalized [ika?] satisfy Max, however, since both have some consonant corresponding to the underlying /t/. Both of these candidates fail CodaCond, so the decision falls to the markedness constraints, which pick [ika?]. MaxPlace is ranked too low to force faithful parsing of the underlying place feature, so the markedness constraints cause the consonant to have the least marked possible Place, resulting in /ikat/ -> [ika?]. The relative rankings of CodaCond and PLHier >> MaxPlace do not matter; we can see this most clearly by comparing (28) to the following tableau, which gets the same result even though CodaCond is low ranked:

\begin{tabular}{|l|c|c|c|c|c|c|}
\hline /ikat/ & Max & Dep & $*$ Cor & $*$ Phar & MaxPl & CodaCon \\
\hline ikat & & & $* !$ & & & $*$ \\
\hline ikati & & $* !$ & $*$ & & & \\
\hline ika & $* !$ & & & & $*$ & \\
\hline 止ika? & & & & $*$ & $*$ & $*$ \\
\hline
\end{tabular}

A language with coda Place neutralization will still be able to maintain a place distinction in onsets. Recall that IDOnsPlace is always above PLHier, and in addition, in this grammar Max is high ranked. Max thus forces syllable-initial consonants to remain, and IDOnsPlace forces them to keep their underlying Place:

(32) Onsets in syllable-final Place neutralization language

\begin{tabular}{|c|c|c|c|c|c|c|c|}
\hline /dakap/ & IDOnsP & Max & Dep & CodCd & ${ }^{*}$ Cor & $*$ Phar & MaxPl \\
\hline dakap & & & & & * & & \\
\hline akap & & $* !$ & & & & & $*$ \\
\hline ?akap & $* !$ & & & & & $*$ & $*$ \\
\hline
\end{tabular}


The high-ranking of IDOnsPlace also means that there will not be languages that avoid coda place while satisfying MaxPlace by moving the coda Place feature to an onset consonant, since this would result in unfaithfulness to the underlying Place of the onset ${ }^{11}$.

\subsection{Deletion: Place and Voice}

Having seen the analysis of Place neutralization we can now account for the difference in Place and Voice with respect to deletion. If Max is ranked below CodaCond, the latter can be satisfied by deletion of the offending consonant:

\begin{tabular}{|c|c|c|}
\hline /pik/ & CodaCond & Max \\
\hline pik & $* !$ & \\
\hline pi & & * \\
\hline
\end{tabular}

The same effect can be caused by the interaction of MaxPlace and CodaCond. In order to satisfy MaxPlace, the consonant must remain, since the Place feature must be associated to a consonant. Thus if MaxPlace outranks CodaCond, the consonant must be retained despite the CodaCond violation.

\begin{tabular}{|l|c|c|}
\hline$/$ pik/ & MaxPlace & CodaCond \\
\hline$\square$ pik & & $*$ \\
\hline pi & $* !$ & \\
\hline
\end{tabular}

Thus, both Max and MaxPlace must be low ranked for deletion to be optimal: the ranking must be Dep,CodaCond >> MaxPlace, Max. In this grammar, the constraints MaxPlace and Max that force the consonant to remain are lowest ranked. CodaCond prevents the consonant from remaining with syllable-final Place, and Dep forbids epenthesis as a way to change the consonant's syllable position. So deletion is optimal: 
(35) Syllable-final deletion language

\begin{tabular}{|l|c|c|c|c|}
\hline /pik/ & Dep & CodaCond & Max & MaxPlace \\
\hline pik & & $* !$ & & \\
\hline 电 & & & $*$ & $*$ \\
\hline piki & $* !$ & & & \\
\hline
\end{tabular}

Why is this result possible, when it is not possible for Voice?

The representational difference between the unmarked consonants is the crucial point here. As we have seen above, neutralization of Place results in a consonant that does have a Place feature. It still violates some Place markedness constraint, albeit a low ranked one. In contrast, we saw in section 3 that the Laryngeally neutralized consonant was truly unmarked: given the assumption of privative Voice, the plain voiced obstruent has no Laryngeal specification at all, and violates no Laryngeal markedness constraint.

This difference has the result that if Max is low ranked, deletion may be the only way to obey the high-ranked constraints: even / // will violate CodaCond (as well as the markedness constraint) since it does have a Place feature in the coda:

\section{(36) CodaCond interaction with Dep, Max}

\begin{tabular}{|l|c|c|c|c|}
\hline$/$ pik/ & CodaCond & Dep & Max & MaxPlace \\
\hline pik & $* !$ & & & \\
\hline pi? & $* !$ & & & $*$ \\
\hline 再i & & & $*$ & $*$ \\
\hline piki & & $* !$ & & \\
\hline
\end{tabular}

This differs from the situation for Voice, where even if Max is low, deletion of the entire consonant will never be optimal. Devoiced [pik] in the following tableau satisfies both *Lar and Max, so no reranking of them will make [pi], with deletion, the winner: 


\section{(37) Effect of Place and Lar representational difference}

\begin{tabular}{|l|c|c|}
\hline /pig/ & $*$ Lar & Max \\
\hline pig & $*$ & \\
\hline pik & & \\
\hline pi & & $*$ \\
\hline
\end{tabular}

\subsection{Comparison between CodaCond and Place markedness}

Now that we have seen the interactions of the Place markedness constraints, one final comparison must be made for completeness. We have seen CodaCond triggering epenthesis, deletion and neutralization in the tableaus above. But we have also seen that there are $* \mathrm{~F}$ and positional faithfulness constraints for Place; and we saw in section 3 that the latter type of constraint was sufficient to trigger Voice alternations.

It is true that deletion and Place neutralization both can have an alternation analysis without CodaCond. We saw in section 5.2 that we could produce Place neutralization by the effects of the ranking PLHier >> MaxPlace even when CodaCond was low ranked:

(38) Place neutralization language: a second possibility

\begin{tabular}{|c|c|c|c|c|c|c|}
\hline /ikat/ & Max & ${ }^{*}$ Cor & *Phar & MaxPl & Dep & CodaCon \\
\hline ikat & & $* !$ & & & & $*$ \\
\hline ika? & & & $*$ & $*$ & & $*$ \\
\hline ika & $* !$ & & & $*$ & & \\
\hline ikati & & $* !$ & & & $*$ & \\
\hline
\end{tabular}

Similarly the ranking IDOnsPlace >> PLHier >> Max, MaxPlace,Dep will give a language with deletion, without the need for CodaCond: 
(39) Deletion languages: a second possibility

\begin{tabular}{|l|c|c|c|c|c|c|}
\hline /pik/ & IDOnsPl & $*$ Dor & $*$ Phar & Max & MaxPlace & Dep \\
\hline pik & & $* !$ & & & & \\
\hline pi? & & & $* !$ & & $*$ & \\
\hline œpi & & & & $*$ & $*$ & \\
\hline piki & & $* !$ & & & & $*$ \\
\hline
\end{tabular}

Deletion is optimal here because all consonants, even $/ \Omega /$, violate a PLHier constraint, and since these outrank Max, deleting the consonant entirely incurs the lowest-ranked violation.

However, recall that Place and Voice alternations differ. These two alternations are possible without CodaCond, but remember the results of section 3: there is never epenthesis to save syllable-final voiced consonants, but there are languages with epenthesis in response to the coda constraint on Place. As shown there, such a grammar cannot be the result of positional faithfulness, but is only possible given the existence of the CodaCond constraint.

\section{Summary: Why different patterns?}

Now that we have seen the analyses of the relevant Place alternations, we can sum up why Place and Voice show different phonological patterns.

For Voice, we made two assumptions. One is that Voice is privative. The other is that the Voice alternations were the result of context-free markedness (*Lar) interacting with positional faithfulness. The following table, repeated from section 3.2, shows the marks incurred by the faithful, devoiced, deleted and epenthesized candidates.

(40) Laryngeal constraints interaction with Dep, Max

\begin{tabular}{|l|l|l|l|l|}
\hline & $*$ Lar & MaxLar & Dep & Max \\
\hline pig & $*$ & & & \\
\hline pik & & $*$ & & \\
\hline pigi & $*$ & & $*$ & \\
\hline pi & & $*$ & & \\
\hline
\end{tabular}


Examining these marks, we see that the only possible grammars will be those where [pig] and [pik] are optimal. [pigi] will never win because its marks are a superset of those incurred by [pig]: Both violate *Lar, so that the additional Dep mark incurred by [pigi] will be decisive wherever it is ranked. Thus, we will be able to construct a ranking where [pig] is optimal, but never one where [pigi] is. Comparing the remaining two candidates, then, we see that a similar relationship holds between [pi] and [pik]. Both violate *MaxLar; thus the additional Max violation incurred by [pi] will always be decisive. So we will be able to construct a ranking where [pik] is optimal, but never one where [pi] is.

Thus, there are only two possible grammars for Laryngeal coda effects: the one where faithful [pig] wins - the languages with no voicing alternations in coda - and the one where [pik] wins - the languages with final devoicing.

The alternations shown by Place restrictions differ for two reasons. One is the fact that there are no truly Placeless consonants. Thus, if Max is low, deletion may be the only way to obey the high-ranked constraints: even / / will violate CodaCond (as well as the markedness constraint) since it does have a Place feature in the coda:

(41) CodaCond interaction with Dep, Max

\begin{tabular}{|l|c|c|c|c|}
\hline$/$ pik/ & CodaCond & Dep & Max & MaxPlace \\
\hline pik & $* !$ & & & \\
\hline pi? & $* !$ & & & $*$ \\
\hline एpi & & & $*$ & $*$ \\
\hline piki & & $* !$ & & \\
\hline
\end{tabular}

This differs from the situation for Voice, where even if Max is low, deletion of the entire consonant will never be optimal. Devoiced [pik] in the following tableau satisfies both *Lar and Max, so no reranking of them will make [pi], with deletion, the winner: 
(42) Effect of Place and Lar representational difference

\begin{tabular}{|l|c|c|}
\hline /pig/ & $*$ Lar & Max \\
\hline pig & $*$ & \\
\hline pik & & \\
\hline pi & & $*$ \\
\hline
\end{tabular}

The other difference between Place and Voice is due to the fact that there is a CodaCond constraint for Place but not for Laryngeal, with the result that epenthesis is only possible in response to Place. A context-free constraint like *Lar cannot result in epenthesis, as it does not treat the epenthesized and non-epenthesized candidates differently. The following table summarizes the marks for the relevant constraints.

(43) Different effects of CodaCond and markedness

a.

\begin{tabular}{|l|c|c|}
\hline$/$ pik/ & CodaCond & Dep \\
\hline pik & $*$ & \\
\hline piki & & $*$ \\
\hline
\end{tabular}

b.

\begin{tabular}{|l|c|c|}
\hline$/$ pik/ & $*$ Dor & Dep \\
\hline pik & $*$ & \\
\hline piki & $*$ & $*$ \\
\hline
\end{tabular}

\begin{tabular}{|l|c|c|}
\hline$/$ pig/ & $*$ Lar & Dep \\
\hline pig & $*$ & \\
\hline pigi & $*$ & $*$ \\
\hline
\end{tabular}

As we see in (a), then, epenthesis may sometimes arise for place features due to the effect of CodaCond, although it cannot be an effect of markedness; but as in (b), since there is no such additional CodaCond constraint for laryngeal features, and markedness constraints alone cannot trigger insertion, there will never be epenthesis to save laryngeal features. Thus the correct patterns are generated for the different features. 
If we now sum up the proposed constraints affecting Place in a single tableau and compare the marks given for the candidates, we see that their relationship is quite different from the one that holds among the Voice candidates.

\begin{tabular}{|l|l|l|l|l|l|l|}
\hline /pik/ & Max & Dep & CodaCon & $*$ Dor & $*$ Phar & MaxPl \\
\hline pik & & & $*$ & $*$ & & \\
\hline piki & & $*$ & & $*$ & & \\
\hline pi & $*$ & & & & & $*$ \\
\hline pi? & & & $*$ & & $*$ & $*$ \\
\hline
\end{tabular}

As is clear, there is no subset relation between the marks of any pair of the candidates in the above tableau. For example, although both [pik] and [pi?] violate CodaCond, they differ in marks incurred for other constraints; thus, depending on the rankings, either may be optimal. So, as we have already seen in section 5, we can construct grammars that will make any one of these candidates optimal. This is the correct result, since each type of language is attested: The types that allow coda Place, that neutralize coda Place, that epenthesize in response to coda Place, and that delete in response to it.

Thus, the resulting typology confirms the correctness of the proposed constraints for both Place and Voice; in both cases we generate all and only the attested languages.

\section{Conclusion}

These results are important for a number of reasons. The specifics of the analysis attest to the importance of the MaxF family of constraints and positional faithfulness constraints, and imply these constraint families will be found for other features. Markedness constraints (the *F family) are used to account for phenomena that formerly were analyzed using underspecification; it is shown that this can be a fruitful way of resolving underspecification paradoxes like the problems with the hypothesis that $/ \mathrm{h}, ? /$ have no Place features.

But a broader point is perhaps even more important. This is not the only case in featural phonology where there are asymmetries in the types of alternations associated with a constraint. This paper has made at least three points regarding the analysis of such asymmetries. 
One is the fact that constraints are not purely formal in nature, but can have substantive differences: just because UG contains Coda Condition on a certain feature does not inevitably mean that it contains a Coda Condition on some other feature - we must demonstrate the existence of proposed constraints in UG by phonological argumentation. As we have seen above, the facts point toward the existence of a Coda Condition for Place, but not one for Voice. $^{12}$

The other line of argument is based on a representational differences, that there are no Placeless consonants, but there are consonants with no Laryngeal features. Thus although I have argued against underspecification of Place, I have also shown that we cannot necessarily retreat to the assumption that representations are always composed of fully specified binary features. Proposals like the privative nature of the feature Voice and the resulting lack of specification of voiceless obstruents still have a role to play in the OT framework.

The general form of the interaction that results from the representational possibilities for Laryngeal features is a significant one. Because *Lar can be satisfied by simply deleting the offending Laryngeal feature, deletion of the whole segment is overkill - it incurs additional unnecessary Max violations. Thus no ranking can make segment deletion an optimal way of satisfying * Lar. Thus we see a type of alternation universally ruled out without any additional stipulation: it falls out from the basic constraints that are needed and from the proposed representations, because of the resulting subset relationship between the marks of the two candidates. It is clear that this type of interaction should be considered as a possible line of attack on other such phonological asymmetries.

Finally, further research is needed to determine the status of IdentF constraints, given the demonstration herein that MaxF constraints are necessary. It may be preferable to have only one family of featural faithfulness constraints if possible, but to replace Ident with MaxF in all situations is not trivial. 


\section{References}

Abbott, Miriam. 1986. Macushi. In Pullum, G. and D. Derbeyshire, eds., Handbook of Amazonian Languages, volume 3. The Hague: Mouton de Gruyter. 23-160.

Beckman, Jill. 1999. Positional faithfulness. PhD dissertation, University of Massachusetts, Amherst.

Beckman, Jill. 1995. Shona height harmony: markedness and positional identity. University of Massachusetts Occasional Papers 18. GLSA, Amherst MA. 53-75.

Bee, Darlene and Kathleen Barker Glasgow. 1973. Usarufa tone and segmental phonemes. In McKaughan, Howard, ed., The languages of the Eastern family of the East New Guinea Highland stock. University of Washington Press. 190-224.

Bessell, Nicola. 1992. Towards a phonetic and phonological typology of post- velar articulation. PhD dissertation, University of British Columbia.

Blevins, Juliette and Andrew Garrett. 1993. The evolution of Ponapeaic nasal substitution. Oceanic Linguistics 32: 199-236.

Carson, Neusa M. 1982. Phonology and morphosyntax of Macuxi (Carib). PhD dissertation, University of Kansas.

Causley, Trisha. 1997. Identity and featural correspondence: the Athapaskan case. Proceedings of NELS 27. Amherst: GLSA. 93-101.

Cho, Young-Mee Y. 1990. Parameters of consonantal assimilation. PhD dissertation, Stanford University.

Cole, Jennifer and Charles Kisseberth. 1994. An optimal domains theory of harmony. Studies in the Linguistic Sciences 24: 101-14.

Eckman, Fred R. 1981. On the naturalness of interlanguage phonological rules. Language Learning 31: 195-216.

Edge, Beverley A. 1991. The production of word-final voiced obstruents by L1 speakers of Japanese and Cantonese. Studies in Second Language Acquisition 13: 377-393.

Fukazawa, Haruka and Viola Miglio. 1998. Restricting conjunction to constraint families. Proceedings of Western Conference on Linguistics 96, volume 9: 102-117.

Gafos, Adamantios and Linda Lombardi. 1997. Consonant transparency and vowel echo. Proceedings of NELS 29.

Ito, Junko. 1989. A prosodic theory of epenthesis. Natural Language and Linguistic Theory 7: 217-59 
Ito, Junko. 1986. Syllable theory in prosodic phonology. $\mathrm{PhD}$ dissertation, University of Massachusetts at Amherst.

Ito, Junko and Armin Mester. 1994. Reflections on CodaCond and Alignment. Phonology at Santa Cruz III, eds. Jason Merchant, Jaye Padgett and Rachel Walker. Santa Cruz: Linguistics Research Center. 27-46 .

Ito, Junko and Armin Mester. 1997. Sympathy theory and German truncations. University of Maryland Working Papers in Linguistics 5: 117-138.

LaMontagne, Greg and Keren Rice. 1995. A Correspondence account of coalescence. University of Massachusetts Occasional Papers 18: 249-384.

Legendre, Geraldine, William Raymond, and Paul Smolensky. 1993. An optimality-theoretic typology of case and grammatical voice systems.

Proceedings of the 19th annual meeting of the Berkeley Linguistic Society. 464-478. Lombardi, Linda. 1991. Laryngeal features and laryngeal neutralization . PhD dissertation, University of Massachusetts, Amherst. Published by Garland, 1994.

Lombardi, Linda. 1994. Postlexical rules and the status of privative features. Phonology 13: $1-38$.

Lombardi, Linda. 1995a. Laryngeal neutralization and syllable wellformedness. Natural Language and Linguistic Theory 13:39-74.

Lombardi, Linda. 1995b. Laryngeal features and privativity. The Linguistic Review 12: 355-59.

Lombardi, Linda 1997. Coronal sonorants and markedness. Maryland Working Papers in Linguistics 5: 156-175

Lombardi, Linda 1999. Positional faithfulness and the phonology of voicing in Optimality Theory. Natural Language and Linguistic Theory 17: 267-302.

McCarthy, John J. 1989. Guttural phonology. Ms., University of Massachusetts, Amherst. McCarthy, John J. 1993. The parallel advantage: containment, consistency, and alignment. Handout of talk presented at Rutgers Optimality Workshop 1.

McCarthy, John J. 1994a. The phonetics and phonology of Semitic pharyngeals. Keating, P. ed., Phonological structure and phonetic form: Papers in Laboratory Phonology III. Cambridge University Press.

McCarthy, John J. 1994b. On [coronal] "transparency." Handout of talk presented at TREND-2. 
McCarthy, John J. and Alan Prince. 1995. Faithfulness and Reduplicative Identity. University of Massachusetts Occasional Papers 18. GLSA, Amherst MA. 249384.

Maryott, Kenneth. 1961. The phonology and morphophonemics of Tabukang Sangir. Phillipine social science and humanties review, 26: 111-26.

Mester, R.A. and Junko Ito. 1989. Feature predictability and underspecification: Palatal prosody in Japanese mimetics. Language 65: 259-93.

Mills, Roger. 1975. Proto South Sulawesi and Proto Austronesian Phonology. PhD dissertation, University of Michigan.

Mithun, M. and H. Basri. 1986. The phonology of Selayarese. Oceanic Linguistics 24: 210254.

Mohanan, K.P. 1992. The emergence of complexity in phonological development. In Ferguson et al eds., Phonological Development: Models, Research, Implications.

Timonium, MD: York Press. 635-62.

Nicholson, Ruth and Ray. 1962. Fore phonemes and their interpretation. Studies in New Guinea Linguistics, Oceanic Linguistics Monographs 6: 128-148.

Okell, John 1969. A reference grammar of colloquial Burmese. London: Oxford University Press.

Padgett, Jaye. 1995a. Partial class behavior and nasal place assimilation. Proceedings of the South Western Optimality Theory Workshop. University of Arizona Coyote Papers, Tucson. 145-183.

Padgett, Jaye. 1995b. Feature classes. University of Massachusetts Occasional Papers 18, GLSA, Amherst MA. 385-420.

Pater, Joe. 1995. Austronesian nasal substitution and other NC effects. In R. Kager, H. van der Hulst, W. Zonneveld, eds. The Prosody Morphology Interface, Cambridge: Cambridge University Press. 310-343.

Payne, David L. 1981. The phonology and morphology of Axininca Campa. Summer Institute of Linguistics, University of Texas at Austin.

Prince, Alan and Paul Smolensky. 1993. Optimality theory: Constraint interaction in generative grammar . To appear, MIT Press.

Rehg, Kenneth L. 1984. Nasal substitution rule in Ponapean. In B.W. Bender, ed., Studies in Micronesian linguistics. Pacific Linguistics series C number 80. 317-337. 
Rehg, Kenneth L. and Damian G. Sohl. 1981. Ponapean Reference Grammar. University of Hawaii Press.

Rice, Keren. 1995. The representation of the perfective suffix in the Athabaskan language family. International Journal of American Linguistics 61: 1-37.

Rice, Keren. 1989. A grammar of Slave. Mouton de Gruyter.

Sapir, J. David. 1965. A grammar of Diola-Fogny. Cambridge University Press.

Smolensky, Paul. 1993. Harmony, markedness and phonological activity. Paper presented at Rutgers Optimality Workshop 1 and revised handout.

Schachter, Paul and Victoria Fromkin. 1968. A phonology of Akan: Akuapem, Asante, Fante. Working Papers in Phonetics 9, UCLA.

Steriade, Donca. 1982. Greek prosodies and the nature of syllabification. $\mathrm{PhD}$ dissertation, MIT.

Steriade, Donca. 1987. Locality conditions and feature geometry. Preceedings of NELS 17. 595-618.

Teoh, Boon Seong. 1988. Aspects of Malay phonology revisted, a non-linear approach. $\mathrm{PhD}$ dissertation, University of Illinois.

Walker, Rachel. 1997. Faith and Markedness in Esimbi Feature Transfer. Phonology at Santa Cruz 5: 103-115.

Waltz, Nathan and Carolyn. 1967. Guanano phonemics. In Waterhouse, Viola, Phonemic systems of Columbian languages. Summer Institute of Linguistics, Norman, OK. 25-36.

Weinberger, Steven H. 1994. Functional and phonetic constraints on second language phonology. In Yavas, Mehmet, ed., First and Second Language Phonology. San Diego: Singular Publishing. 283-302.

Weinberger, Steven H. 1987. The influence of linguistic context on syllable simplification. In Ioup, Georgette and Steven Weinberger, eds., Interlanguage Phonology: The acquisition of a second language sound system. Cambridge: Newbury House Publishers. 401-417.

West, Birdie and Betty Welch. 1967. Phonemic system of Tucana. In Waterhouse, Viola, Phonemic systems of Columbian languages. Summer Institute of Linguistics, Norman, OK. 11-24.

Young, Rosemary. 1962. The phonemes of Kanite, Kamano, Benabena and Gahuku. Studies in New Guinea Linguistics, Oceanic Linguistics Monographs 6. 90-110. 
* Thanks to Jill Beckman, Jaye Padgett, Joe Pater, Alan Prince, Keren Rice, Paul Smolensky, and especially John McCarthy, and to audiences at Rutgers, University of Massachusetts at Amherst, University of Pennsylvania, Johns Hopkins, and my graduate classes at the University of Maryland. Thanks also to several reviewers whose comments were useful and apologies to anyone whose assistance during the long life of the manuscript I may have omitted.

1. Interlanguage sometimes shows a pattern of syllable simplification where final voiced consonants trigger epenthesis and final voiceless do not, when the native language does not allow final voiced consonants (Edge 1991, Eckman 1981). Eckman (1981) notes that no such pattern occurs in natural languages and uses this as support to argue that interlanguages are not necessarily natural languages. As several researchers have noticed, there is a strong effect of experimental task (Eckman 1981, Weinberger 1987, 1994, Edge 1991): epenthesis is found with elicitation by reading of word lists, whereas in spontaneous speech there tends to be deletion of all syllable-final consonants and/or final devoicing of voiced obstruents.

2. I assume that in an OT framework this will be due to an overriding right-alignment constraint as in McCarthy (1993) or RightAnchor as in McCarthy and Prince (1995; although see Lombardi 1999 for a different approach to a similar type of pattern for laryngeal features). The restrictions on nasal substitution include that it only applies to clusters with identical Place, clearly reflecting the importance of faithfulness to Place. It does not apply to coronal clusters for various reasons. For further details see the Rehg references, Ito (1986), Lombardi (1994), Blevins and Garrett (1993).

3. Again, word-final consonants are unaffected (see previous footnote). Note that for Diola the coda constraint is on [+cons] in Ito 1986, but this language is listed as a case of the coda filter in Ito 1989, which does not appear to recognize any but Place coda filter. Diola also has epenthesis when total reduplication takes place and possibly in other morphological situations; the source is unclear on further details, saying only "Different grammatical situations call for one or the other."

4. Lombardi (1999) uses IdentVoice for the general (non-positional) faithfulness constraint. This results in a very minor difference in predictions for the cross-linguistic typology of voicing assimilation and neutralization, which is of no concern for the issues in this paper. 
5.There is a phenomenon in Slave that is an apparent exception to this generalization unless we are attentive to the details of Rice's (1995) analysis. As seen in section 2.2.3, Slave has syllablefinal debuccalization. We can see this in imperfective stems ([1] is a voiced lateral fricative):

With V-initial suffix Syllable-final form

$$
\text { -ji[1]e -ji[h] 'breathe' }
$$

Perfective forms of the same stems show a different alternation: the voiced fricative deletes in the syllable-final form: -ji 'breathe, perf.' Superficially this looks like deletion of impermissible syllable-final voiced consonants, but it is clearly not as simple as that. We see different effects in different morphological forms of the same verb even though the phonological environment syllable-final - is the same. Thus this cannot be simply a phonological rule deleting all syllablefinal voiced consonants, but must have some additional phonological or morphological conditioning that only occurs in the perfective. Rice's account is that there is a underspecified perfective suffix in Athabaskan, and that this is a phonological effect resulting from the illformedness of the resulting consonant when a voiced fricative is fused with the suffix; the resulting ill-formed consonant is what is subject to deletion, or more precisely, it is impossible to phonetically realize it (Rice p24). Thus this is not a general process deleting syllabically impermissible voiced consonants, but rather deletion of a particular voiced segment which is ruled out generally in the language. (Thanks to Keren Rice for pointing out this example.)

6.Ito and Mester (1994) propose in an OT framework that CodaCond should be stated as a positive constraint requiring left alignment of CPlace and the syllable edge; they also discuss how to formulate the double linking effects. As far as I can tell, either a negative formulation as in Ito $(1986,1989)$ or the Ito and Mester (1994) formulation will interact in the same way with the constraints I will discuss. I call the constraint CodaCond, rather than using an alignment formulation, for the sake of familiarity.

7. If we assume a MaxPlace constraint (rather than IdentPlace) parallel to MaxVoice, either high ranking of Max or of MaxPlace will give the result that epenthesis is optimal. This may seem like a redundancy, but the different rankings may in fact give different results elsewhere. We might see differences in circumstances of assimilation, where Place changes but there is no segment deletion; ghost consonants might show differences between Max violations and MaxPlace violations, if Max does not apply to a ghost since it is not a full segment (Zoll this volume). One might also consider, in this context, whether it is necessarily the case that all speakers of a given language in fact have identical grammars; for some discussion see Mohanan 
(1992).

8. Although the facts lead inexorably to this conclusion, we might ask, why does such a constraint exist for Place and not Voice? I am unable at this point to give a simple explanation, but perhaps this is not unexpected given fundamental differences between Place and Voice. It is truly marked to have laryngeal features: many languages have only voiceless unaspirated consonants. On the contrary, it is not marked in the same way for a consonant to have a Place feature: rather it is the normal situation for a consonant to have Place. Place is a fundamental requirement for being a consonant and realizing other consonantal features such as stricture. And all languages have Place distinctions: unlike the case with Laryngeal distinctions, there is no language where all consonants have the unmarked Place. Certainly particular Places differ in their respective markedness, but it is not the case that simply to have Place is a marked situation, for a consonant; however it is marked to have Place in a coda, and CodaCond expresses this.

9. Note that while I am adopting McCarthy's features, I am not necessarily assuming his oral/pharyngeal node feature geometry as well, given Padgett's (1995b) convincing arguments for using feature classes rather than class nodes in OT. In addition to languages that show the guttural natural class (Phar), there are Salish languages where we see a natural class of uvulars and pharyngeals excluding the laryngeals (Bessell 1992). The proposed features also allow this, as this is the class [-glottal].

10. See Smolensky (1993), Cole and Kisseberth (1994), McCarthy (1994b), and Ní Chiosáin and Padgett (this volume) for different accounts of transparency effects that also do not require underspecification.

11. A remaining possible typological problem is that if both Max and MaxPlace are ranked below the *F constraints on Place, we can generate an unattested language that deletes all syllable-initial consonants. This may be an accidental gap, since the resulting language has obvious functional problems. However, it seems possible that this problem only exists because we have incorrectly defined the onset faithfulness constraints. If the IdentOnsPlace constraint were replaced by MaxOnsPlace and DepOnsPlace, deleted onsets would violate universally high-ranking MaxOnsPlace. and such a language would no longer be predicted. For reasons of space I do not pursue this option here. 
12. Smolensky has suggested in presentations that the Coda Condition should be analyzed as a conjunction of the more basic constraints *Place and NoCoda. This predicts that we could also construct the constraint *Voice \& NoCoda and use it to account for coda Voice effects, and this has in fact been done by Ito and Mester (1997, see also this volume). The latter is equivalent to a coda condition on Voice, which as I have shown in this paper makes incorrect predictions. This suggests that we should rule out conjunctions of this type. Fukazawa and Miglio (1998; see also Fukazawa this volume) propose we restrict conjunction to constraints within the same constraint family, which would rule out such a conjunction; the question remains how we would then account for the results of Ito and Mester (this volume). 